\title{
ON BOUNDARY VALUE PROBLEMS FOR DEGENERATE DIFFERENTIAL INCLUSIONS IN BANACH SPACES
}

\author{
VALERI OBUKHOVSKII AND PIETRO ZECCA
}

Received 21 December 2002

We consider the applications of the theory of condensing set-valued maps, the theory of set-valued linear operators, and the topological degree theory of the existence of mild solutions for a class of degenerate differential inclusions in a reflexive Banach space. Further, these techniques are used to obtain the solvability of general boundary value problems for a given class of inclusions. Some particular cases including periodic problems are considered.

\section{Introduction}

In the last decades the theory of degenerate differential equations in Banach spaces attracted the attention of a large number of researchers (see, e.g., Favini and Yagi [5], Showalter [13], and [6] and the references therein). One of the main reasons is that many partial differential equations arising in mathematical physics and in applied sciences may be naturally presented in this form.

In this paper, we introduce a class of degenerate differential inclusions in a reflexive Banach space and define the notion of mild solution for such inclusion. Applying the techniques of the theory of condensing multimaps (see Kamenskii et al. [8]) and the methods of the theory of multivalued linear operators [3, 5 ], we first prove existence results for the Cauchy problem for various types of multivalued nonlinearities. Then we consider the solvability of general boundary value problems and discuss some particular cases including periodic problem. It should be noted that, starting from the paper of Zecca and Zezza [15], nonlinear boundary value problems for nondegenerate differential inclusions in Banach spaces were studied in a number of papers (see, e.g., $[4,9,10,11,12]$ ) under compactness conditions on the evolution operator generated by the linear part of the problem. In our paper we do not suppose compactness for the linear part nor for the multivalued nonlinearity. We only assume some regularity conditions expressed in terms of the measures of noncompactness. 


\section{Preliminaries}

2.1. Multivalued linear operators. We present some necessary definitions and results from the theory of multivalued linear operators. Details can be found in $[3,5]$.

Let $E$ be a complex Banach space.

Definition 2.1. A multivalued map (multimap) $A: E \rightarrow 2^{E}$ is said to be a multivalued linear operator (MLO) in $E$ if

(1) $D(A)=\{x \in E: A x \neq \varnothing\}$ is a linear subspace of $E$;

(2) the following linearity relations hold:

$$
\begin{gathered}
A x+A y \subset A(x+y), \quad \forall x, y \in D(A), \\
\lambda A x \subseteq A(\lambda x), \quad \forall \lambda \in \mathbb{C}, x \in D(A) .
\end{gathered}
$$

It is an easy consequence of the definition to note that $A x+A y=A(x+y)$ for all $x, y \in D(A)$ and $\lambda A x=A(\lambda x)$ for all $x \in D(A), \lambda \neq 0$.

Definition 2.2. The inverse $A^{-1}$ of an MLO is defined as

(1) $D\left(A^{-1}\right)=R(A)$;

(2) $A^{-1} y=\{x \in D(A): y \in A x\}$.

It is easy to verify that $A^{-1}$ is an MLO in $E$.

Definition 2.3. Let $A$ and $B$ be two MLOs in $E$. The sum and the product of $A$ and $B$ are defined, respectively, by the relations

$$
\begin{gathered}
D(A+B)=D(A) \cap D(B), \quad(A+B) x=A x+B x, \\
D(A B)=\{x \in D(B): D(A) \cap B x \neq \varnothing\}, \quad A B x=A(D(A) \cap B x) .
\end{gathered}
$$

One can observe that $A+B$ and $A B$ are MLOs in $E$ and that

$$
(A B)^{-1}=B^{-1} A^{-1} \text {. }
$$

Definition 2.4. The resolvent set $\rho(A)$ of an MLO $A$ is defined as the collection of all $\lambda \in \mathbb{C}$ for which

(1) $R(\lambda I-A)=D\left((\lambda I-A)^{-1}\right)=E$;

(2) $(\lambda I-A)^{-1}$ is a single-valued bounded operator on $E$.

The operator $(\lambda I-A)^{-1}$ is called the resolvent of $A$.

Definition 2.5. An MLO A satisfies the Hille-Yosida condition $(\mathrm{H}-\mathrm{Y})$ if

(i) the resolvent set $\rho(A)$ contains an interval $(\beta,+\infty),-\infty<\beta<+\infty$;

(ii) the resolvent $(\lambda I-A)^{-1}$ satisfies the estimates

$$
\left\|(\lambda I-A)^{-n}\right\|_{\mathscr{L}(E)} \leq \frac{C}{(\lambda-\beta)^{n}}, \quad \lambda>\beta, n=1,2, \ldots,
$$

for some positive constant $C$. 
The following statement gives an example of MLO satisfying $(\mathrm{H}-\mathrm{Y})$. Let $E^{*}$ denote a space dual to $E$ and $J: E \rightarrow 2^{E^{*}}$ be the duality map.

Proposition 2.6. Given an $M L O A$, suppose that for every $x \in D(A)$ there exists $x^{*} \in J(x)$ such that

$$
\operatorname{Re}\left\langle y, x^{*}\right\rangle \leq \beta\|x\|^{2}
$$

for all $y \in A x$, where $\beta$ is a real number. Let also

$$
R\left(\lambda_{0} I-A\right)=E
$$

for some $\lambda_{0}>\beta$. Then $(\beta,+\infty) \subset \rho(A)$ and the resolvent $(\lambda I-A)^{-1}$ satisfies (ii) of condition $(H-Y)$ with $C=1$.

The proof follows the lines of [5, Theorem 2.7].

Let $A$ be an MLO satisfying (H-Y).

Definition 2.7. For an integer $n>\beta$, the bounded linear operator

$$
A_{n}=n\left[-I+n(n I-A)^{-1}\right]
$$

is called a Yosida approximation of $A$.

Denote by $\exp \left\{t A_{n}\right\}$ the semigroups generated by $A_{n}, n>\beta$.

Proposition 2.8 (see [5, Section 2.1]). Let $E$ be a reflexive Banach space and $A$ an $M L O$ on $E$ satisfying $(H-Y)$. The space $E$ can be represented as $E=E_{0} \oplus E_{1}$, where $E_{0}=\overline{D(A)}$ and $E_{1}=A 0$. The sequence $\exp \left\{t A_{n}\right\}, t>0$, converges strongly to a bounded linear operator $\exp \{t A\} \in \mathscr{L}(E)$ which defines a semigroup on $E$ with the estimate

$$
\|\exp \{t A\}\| \leq C e^{\beta t}
$$

The restriction of $\exp \{t A\}$ on $E_{0}$ defines a $C_{0}$-semigroup on $E_{0}$ and the restriction on $E_{1}$ vanishes for $t>0$.

Definition 2.9. Let $P: E \rightarrow E_{0}$ be the projection; we will say that the strongly continuous operator-valued function $U:[0,+\infty) \rightarrow \mathscr{L}(E), U(t) x=\exp \{t A\} P x$, is a generalized semigroup generated by the MLO $A$.

2.2. Degenerate differential inclusions. Let $E$ be a real reflexive Banach space and let $M$ and $L$ be single-valued linear operators on $E$ satisfying the condition

$(\mathrm{ML}) D(L) \subseteq D(M)$ and $\overline{M(D(L))} \subseteq R(M)$.

Let $F:[0, T] \times E \multimap E$ be a given multimap. 
Consider the following Cauchy problem for a degenerate differential inclusion of the form

$$
\begin{aligned}
& \frac{d M x(t)}{d t} \in L x(t)+F(t, M x(t)), \quad t \in[0, T], \\
& M x(0)=y_{0} \in \overline{M(D(L)) .}
\end{aligned}
$$

With the change $y(t)=M x(t)$, we can rewrite problem (2.9) in the form

$$
\begin{gathered}
\frac{d y}{d t} \in A y(t)+F(t, y(t)), \quad t \in[0, T], \\
y(0)=y_{0},
\end{gathered}
$$

where $A=L M^{-1}$. It is clear that $A$ is an MLO operator if $M$ is noninvertible.

We suppose that $A$ satisfies the (H-Y) condition.

Definition 2.10. A function $x:[0, T] \rightarrow E$ is a mild solution of problem (2.9) if the function $M x$ has the form

$$
M x(t)=U(t) M x(0)+\int_{0}^{t} U(t-s) f(s) d s,
$$

where $f \in L^{1}((0, T) ; E)$ is a measurable selection of the multifunction $t \multimap F(t$, $M x(t))$.

The definition is motivated by the following facts. First of all, following [5, Theorem 2.6], it is easy to verify that given a function $f \in L^{1}((0, T) ; E)$, every Carathéodory solution to the problem

$$
\frac{d y(t)}{d t} \in A y(t)+f(t), \quad y(0)=y_{0} \in D(A)
$$

is necessarily of the form

$$
y(t)=U(t) y_{0}+\int_{0}^{t} U(t-s) f(s) d s .
$$

Furthermore, the function

$$
t \longrightarrow U(t) y_{0}+\int_{0}^{t} U(t-s) f(s) d s, \quad y_{0} \in \overline{D(A)}
$$

takes its values in the subspace $\overline{D(A)}=\overline{M(D(L))} \subseteq R(M)$ (see condition (ML)). At last, in the nondegenerate case $M=I$, the given definition agrees with the notion of mild solution for a semilinear differential inclusion (see, e.g., [8]).

To present sufficient conditions under which the MLO $A=L M^{-1}$ satisfies $(\mathrm{H}-\mathrm{Y})$, we recall that, in the Banach space $E$, a semiscalar product can be defined as $[u, v]=\left\langle u, v^{*}\right\rangle$ with $v^{*} \in J(v)$ (see [14]). 
Proposition 2.11. Suppose that

(a) $[L x, M x] \leq \beta\|M x\|^{2}$ for all $x \in D(L)$ for some real $\beta$;

(b) $R\left(\lambda_{0} M-L\right)=E$ for some $\lambda_{0}>\beta$.

Then the MLO A $=L M^{-1}$ satisfies $(H-Y)$ with $C=1$.

This statement follows directly from Proposition 2.6.

2.3. Multimaps and measures of noncompactness. Let $X$ be a metric space and $Y$ a normed space. Let $P(Y)$ denote the collection of all nonempty subsets of $Y$. We denote

$$
\begin{aligned}
K(Y) & =\{S \in P(Y): S \text { is compact }\}, \\
K v(Y) & =\{S \in K(Y): S \text { is convex }\} .
\end{aligned}
$$

We recall some notions (see, e.g., [8] for further details).

Definition 2.12. A multivalued map (multimap) $\mathscr{F}: X \rightarrow P(Y)$ is

(a) upper semicontinuous (u.s.c.) if $\mathscr{F}^{-1}(V)=\{x \in X: \mathscr{F}(x) \subset V\}$ is an open subset of $X$ for every open set $V \subset Y$;

(b) lower semicontinuous (1.s.c.) if $\mathscr{F}^{-1}(W)$ is a closed subset of $X$ for every closed set $W \subset Y$.

Definition 2.13. Let $\mathscr{E}$ be a Banach space and $(\mathscr{A}, \geq)$ a partially ordered set.

A function $\beta: P(\mathscr{E}) \rightarrow \mathscr{A}$ is called a measure of noncompactness (MNC) in $\mathscr{E}$ if

$$
\beta(\overline{\mathrm{CO}} \Omega)=\beta(\Omega) \quad \text { for every } \Omega \in P(\mathscr{E})
$$

A MNC $\beta$ is called

(i) monotone if $\Omega_{0}, \Omega_{1} \in P(\mathscr{E})$ and $\Omega_{0} \subseteq \Omega_{1}$ implies $\beta\left(\Omega_{0}\right) \leq \beta\left(\Omega_{1}\right)$;

(ii) nonsingular if $\beta(\{a\} \cup \Omega)=\beta(\Omega)$ for every $a \in \mathscr{E}$ and $\Omega \in P(\mathscr{E})$;

(iii) invariant with respect to reflection through the origin if $\beta(-\Omega)=\beta(\Omega)$ for every $\Omega \in P(\mathscr{E})$;

(iv) semiadditive if $\beta\left(\Omega_{0} \cup \Omega_{1}\right)=\max \left\{\beta\left(\Omega_{0}\right), \beta\left(\Omega_{1}\right)\right\}$ for every $\Omega_{0}, \Omega_{1} \in$ $P(\mathscr{E})$.

If $\mathscr{A}$ is a cone in $\mathscr{E}$, we say that the MNC $\beta$ is

(v) algebraically semiadditive if $\beta\left(\Omega_{0}+\Omega_{1}\right) \leq \beta\left(\Omega_{0}\right)+\beta\left(\Omega_{1}\right)$ for every $\Omega_{0}$, $\Omega_{1} \in P(\mathscr{E})$;

(vi) regular if $\beta(\Omega)=0$ is equivalent to the relative compactness of $\Omega$.

As an example of MNC satisfying all the above properties, we can consider the Hausdorff MNC

$$
\chi(\Omega)=\inf \{\varepsilon>0: \Omega \text { has a finite } \varepsilon \text {-net }\}
$$


Another example can be presented by the following MNC $\phi$ defined on the space of continuous functions $C([0, T] ; E)$ with the values in a Banach space $E$ :

$$
\phi(\Omega)=\sup _{t \in[0, T]} \chi_{E}(\Omega(t))
$$

where $\chi_{E}$ is the Hausdorff MNC in $E$ and $\Omega(t)=\{y(t): y \in \Omega\}$. The MNC $\phi$ satisfies all the above-mentioned properties except regularity. It is known (see [8]) that for every set $\Omega \subset C([0, T] ; E)$,

$$
\phi(\Omega) \leq \chi_{C}(\Omega)
$$

where $\chi_{C}$ is the Hausdorff MNC in $C([0, T] ; E)$. Moreover, $\phi$ coincides with $\chi_{C}$ on equicontinuous sets $\Omega$ (see [8]).

Let $\mathscr{E}$ and $\mathscr{E}^{\prime}$ be Banach spaces with MNCs $\beta$ and $\beta^{\prime}$, respectively, and let $N: \mathscr{E} \rightarrow \mathscr{E}^{\prime}$ be a bounded linear operator.

Definition 2.14 (see [1]). The value

$$
\|N\|^{\left(\beta, \beta^{\prime}\right)}=\inf \left\{C: \beta^{\prime}(N \Omega) \leq C \beta(\Omega), \Omega \subset \mathscr{E} \text { is a bounded set }\right\}
$$

is called the $\left(\beta, \beta^{\prime}\right)$-norm of $N$.

In particular, if $\beta$ and $\beta^{\prime}$ are the Hausdorff MNCs $\chi$ and $\chi^{\prime}$, then the value $\|N\|\left(\chi, \chi^{\prime}\right)$ is denoted by $\|N\|(\chi)$ and is called the $\chi$-norm of $N$. The $\chi$-norm may be evaluated by the formula (see [1])

$$
\|N\|^{(\chi)}=\chi^{\prime}(N S)=\chi^{\prime}(N B)
$$

where $S$ is a unit sphere and $B$ is a unit ball in $\mathscr{E}$. The above formula easily implies

$$
\|N\|^{(\chi)} \leq\|N\|
$$

Definition 2.15. A multimap $F: X \subseteq \mathscr{E} \rightarrow K(\mathscr{E})$ or a family of multimaps $G$ : $[0,1] \times X \rightarrow K(\mathscr{E})$ is called condensing relative to a MNC $\beta$ (or $\beta$-condensing) if for every set $\Omega \subseteq X$ not relatively compact,

$$
\beta(F(\Omega)) \not \equiv(\Omega) \quad \text { or } \quad \beta(G([0,1] \times \Omega)) \not \equiv \beta(\Omega),
$$

respectively.

Let $W \subset \mathscr{E}$ be an open set, $\mathscr{K} \subseteq \mathscr{E}$ a closed convex subset, $\beta$ a monotone MNC in $\mathscr{E}$, and $F: \bar{W}_{\mathscr{K}} \rightarrow K v(\mathscr{K})$ an u.s.c. multimap such that $x \notin F(x)$ for all $x \in$ $\partial W_{\mathscr{K}}$, where $\bar{W}_{\mathscr{K}}$ and $\partial W_{\mathscr{K}}$ denote, respectively, the closure and the boundary of the set $W_{\mathscr{K}}=W \cap \mathscr{K}$ in the relative topology of the space $\mathscr{K}$.

In such a setting, the relative topological degree $\operatorname{deg}_{\mathscr{K}}(F, \bar{W})$ satisfying the standard properties is defined (see [8, Chapter 3]). 


\section{Existence results}

3.1. Cauchy problem. We consider the Cauchy problem (2.9) under the assumptions that the operators $M$ and $L$ satisfy condition (ML) and $A=M L^{-1}$ satisfies condition ( $\mathrm{H}-\mathrm{Y})$.

On the multivalued nonlinearity $F:[0, T] \times E \multimap E$, we first suppose the following assumptions:

(F0) $F$ has nonempty, compact, and convex values;

(F1) the multifunction $F(\cdot, x):[0, T] \rightarrow K v(E)$ has a strongly measurable selection for every $x \in E$;

(F2) the multimap $F(t, \cdot): E \rightarrow K v(E)$ is u.s.c. for a.a. $t \in[0, T]$;

(F3) there exists a function $\alpha(\cdot) \in L_{+}^{1}([0, T])$ such that

$$
\|F(t, x)\|:=\sup \{\|z\|: z \in F(t, x)\} \leq \alpha(t)(1+\|x\|) \quad \text { for a.a. } t \in[0, T] ;
$$

(F4) there exists a function $k(\cdot) \in L_{+}^{1}([0, T])$ such that

$$
\chi(F(t, D)) \leq k(t) \chi(D), \quad \text { for a.a. } t \in[0, T]
$$

for every bounded set $D \subset E$.

Theorem 3.1. Under conditions (ML), (H-Y), (F0), (F1), (F2), (F3), and (F4), the set $\Sigma$ of all mild solutions of problem (2.9) is nonempty. Moreover, the set $M \Sigma=$ $\{y \in C([0, T] ; E): y(t)=M x(t), x \in \Sigma\}$ is compact.

Proof. From assumptions (F0), (F1), (F2), and (F3), it follows that the superposition multioperator $\mathscr{P}_{F}: C([0, T] ; E) \multimap L^{1}([0, T] ; E)$ given by

$$
\mathscr{P}_{F}(y)=\left\{f \in L^{1}([0, T] ; E): f(t) \in F(t, y(t)) \text { a.e. } t \in[0, T]\right\},
$$

is correctly defined (see [8]), so we can define an integral multioperator $\Gamma$ : $C([0, T], E) \multimap C([0, T], E)$ as

$$
\Gamma y=\left\{z: z(t)=U(t) y_{0}+\int_{0}^{t} U(t-s) f(s) d s: f \in \mathscr{P}_{F}(y)\right\} .
$$

To prove the theorem, it is sufficient to verify that the fixed-point set Fix $\Gamma$ is nonempty and compact.

Consider the closed, convex set $\mathscr{K}=\left\{y: y(0)=y_{0}\right\} \subset C([0, T] ; E)$ and the parametrized family of multioperators $\tilde{\Gamma}: \mathscr{K} \times[0,1] \multimap \mathscr{K}$ such that

$$
\widetilde{\Gamma}(y, \lambda)=\left\{z: z(t)=U(t) y_{0}+\lambda \int_{0}^{t} U(t-s) f(s) d s: f \in \mathscr{P}_{F}(y)\right\} .
$$

Following [8] it is easy to verify that the multimap $\widetilde{\Gamma}$ has compact, convex values; it is u.s.c. and $\nu$-condensing on bounded sets of $\mathcal{K}$, where $\nu$ is the MNC 
in $C([0, T] ; E)$ with values in the naturally partially ordered $\mathbb{R}_{+}^{2}$, defined as

$$
\nu(\Omega)=\max _{\mathscr{D} \subset \Delta(\Omega)}\left(\gamma(\mathscr{D}), \bmod _{C}(\mathscr{D})\right),
$$

where $\Delta(\Omega)$ is the collection of all denumerable subsets of $\Omega$,

$$
\gamma(\mathscr{D})=\sup _{t \in[0, T]} e^{-b t} \chi(\mathscr{D}(t))
$$

given by $b>0$ large enough and $\bmod _{C}(\mathscr{D})$ is the modulus of equicontinuity of D) defined as

$$
\bmod _{C}(\mathscr{D})=\lim _{\delta \rightarrow 0} \sup _{y \in \mathscr{D}} \max _{\left|t_{1}-t_{2}\right| \leq \delta}\left\|y\left(t_{1}\right)-y\left(t_{2}\right)\right\|
$$

Furthermore, using condition (F3) and the standard technique based on the Gronwall-type inequality, it is possible to prove that the set of solutions of the family of inclusions

$$
y \in \widetilde{\Gamma}(y, \lambda)
$$

is a priori bounded in norm by the constant

$$
r_{0}=R\left(\left\|y_{0}\right\|+a\right) e^{R a}
$$

where

$$
a=\int_{0}^{T} \alpha(s) d s, \quad R=\sup _{t \in[0, T]}\|U(t)\| .
$$

Hence, if we take $W$ as an open ball in $C([0, T] ; E)$ centered at the origin with radius $r>r_{0}$, from the basic properties of the topological degree, we have

$$
\operatorname{deg}_{\mathscr{K}}(\Gamma, \bar{W})=\operatorname{deg}_{\mathscr{K}}(\widetilde{\Gamma}(\cdot, 1), \bar{W})=\operatorname{deg}_{\mathscr{K}}(\widetilde{\Gamma}(\cdot, 0), \bar{W})=1,
$$

and the result follows.

We now consider the case when the nonlinearity $F:[0, T] \times E \rightarrow K(E)$ has nonconvex values, but instead of upper Carathéodory conditions (F1) and (F2), it satisfies the almost lower semicontinuity assumption that

$\left(\mathrm{F}_{L}\right)$ there exists a sequence of disjoint compact sets $\left\{I_{n}\right\}, I_{n} \subseteq[0, T]$, such that

(i) $\operatorname{meas}([0, T] \backslash I)=0$, where $I=\cup_{n} I_{n}$;

(ii) the restriction of $F$ on each set $I_{n} \times E$ is l.s.c.

We also assume that the space $E$ is separable.

Theorem 3.2. Under conditions $(M L),(H-Y),\left(F_{L}\right),(F 3)$, and (F4), there exists a mild solution of problem (2.9). 
Proof. We only give a sketch of the proof. In this situation, the superposition multioperator $\mathscr{P}_{F}(x)$ is correctly defined and it is l.s.c.; hence, by FryszkowskiBressan-Colombo theorem (see, e.g., [2]), it admits a continuous selection $\rho(x)$. The same arguments used in the proof of [8, Theorem 5.5.1] imply that there exists a compact convex subset $X \subset C([0, T] ; E)$ invariant with respect to the action of the integral multioperator $\Gamma$. The continuous map $\gamma: X \rightarrow X$ defined by

$$
\gamma(x)(t)=U(t) y_{0}+\int_{0}^{t} U(t-s) \rho(x)(s) d s
$$

is clearly a continuous selection of $\Gamma$. The application of the Schauder fixed-point theorem to $\gamma$ proves the statement.

3.2. Boundary value problems. Let $E$ be a real separable reflexive Banach space. Under hypotheses (ML), (H-Y), (F0), (F1), (F2), (F3), and F(4), we consider the following general boundary value problem:

$$
\begin{gathered}
\frac{d M x(t)}{d t} \in L x(t)+F(t, M x(t)), \quad t \in[0, T], \\
\mathscr{B}(M x)=\mathscr{C}(M x),
\end{gathered}
$$

where $\mathscr{B}: C([0, T] ; E) \rightarrow E$ is a bounded linear operator and $\mathscr{C}: C([0, T] ; E) \rightarrow E$ is a nonlinear (in general) completely continuous operator.

We call generalized Cauchy operator a bounded linear operator $G: L^{1}([0, T] ; E)$ $\rightarrow C([0, T] ; E)$ defined as

$$
(G f)(t)=\int_{0}^{t} U(t-s) f(s) d s
$$

Consider the linear operator $D: C([0, T] ; E) \rightarrow C([0, T] ; E)$ defined as

$$
D(x)(t)=x(t)-U(t) x(0)
$$

and denote $\mathscr{B}_{0}=\mathscr{B}_{\mid \operatorname{Ker}(D)}$. Our main assumption on operators $\mathscr{B}$ and $\mathscr{C}$ is the following:

$(\mathscr{B} \mathscr{C})$ there exists a bounded linear operator $\Lambda: E \rightarrow \operatorname{Ker} D$ such that

$$
\left(I-\mathscr{B}_{0} \Lambda\right)\left(\mathscr{C} y-\mathscr{B}_{B} f\right)=0
$$

for all $y \in C([0, T] ; E)$ and $f \in \mathscr{P}_{F}(y)$. 
We introduce the multioperator $Q: C([0, T] ; E) \multimap C([0, T] ; E)$ defined in the following way:

$$
Q(y)=\Lambda \mathscr{b} y+(I-\Lambda \mathscr{B}) G \mathscr{P}_{F}(y)
$$

(see [15]).

It is easy to see that the fixed points of $Q$ coincide with the functions $y(\cdot)$ defined by the equality $y(t)=M x(t)$, where $x(\cdot)$ is a mild solution of problem (3.14) and (3.15).

To study further properties of the multioperator $Q$, we recall some known facts from [8].

Proposition 3.3. Let $S: L_{1}([0, T] ; E) \rightarrow C([0, T] ; E)$ be an abstract operator satisfying the following conditions:

$\left(\mathrm{S}^{\prime}\right)$ there exists $K \geq 0$ such that

$$
\|S f-S g\|_{C} \leq K\|f-g\|_{L^{1}}, \quad \forall f, g \in L_{1}([0, T] ; E)
$$

(S2) for any compact set $X \subset E$ and sequence $\left\{f_{n}\right\} \subset L_{1}([0, T], E)$ such that $\left\{f_{n}(t)\right\} \subset X$ for a.a. $t \in[0, T]$, the weak convergence $f_{n} \rightarrow f_{0}$ implies $S f_{n}$ $\rightarrow S f_{0}$.

If $\mathscr{P}_{F}$ is a superposition multioperator generated by a multimap $F$ satisfying (Fo), (F1), (F2), (F3), and (F4), then the composition $S \mathscr{P}_{F}$ is an u.s.c. multimap with compact values.

Following the lines of [8, Lemma 4.2.1], it is possible to verify the following proposition.

Proposition 3.4. The generalized Cauchy operator $G$ satisfies properties $\left(S 1^{\prime}\right)$ and (S2).

Since $(I-\Lambda \mathscr{B})$ is a bounded linear operator, we obtain as an immediate consequence the following proposition.

Proposition 3.5. The multioperator $Q$ is u.s.c. and has compact convex values.

Our goal is to give conditions under which the multioperator $Q$ is condensing on bounded subsets of $C([0, T] ; E)$.

We assume that the linear operator $\mathscr{B}$ satisfies the following condition:

$(\mathscr{B})$ the operator $\mathscr{B}$ is $\left(b, \phi, \chi_{E}\right)$-bounded, that is, there exists a constant $b \geq 0$ such that for every bounded set $\Omega \subset C([0, T] ; E)$ we have that

$$
\chi_{E}(\mathscr{B} \Omega) \leq b \phi(\Omega)
$$

where $\phi$ is the MNC in $C([0, T] ; E)$ defined by $(2.18)$. 
We further suppose that the linear operators $U(t)$ satisfy the following estimate for the $\chi$-norm:

$$
\|U(t)\|^{(\chi)} \leq h(t)
$$

where $h:[0, T] \rightarrow \mathbb{R}_{+}$is a summable function.

At last, let the following condition holds:

(C) $\left(1+b\|\Lambda\|^{\left(\chi_{E}, \phi\right)}\right) \sup _{0 \leq t \leq T} \int_{0}^{t} h(t-s) k(s) d s=\mu<1$, where $k(\cdot)$ is the function from condition (F4).

Note that condition (C) is satisfied when the generalized semigroup $U(t)$ is compact $(h(\cdot)=0)$ or when $F$ is completely u.s.c. in the second argument (in the sense that $k(\cdot)=0$ ).

Consider the MNC

$$
\varphi(\Omega)=\left(\phi(\Omega), \bmod _{C}(\Omega)\right)
$$

on the space $C([0, T] ; E)$ with values in the cone $\mathbb{R}_{2}^{+}$, where $\bmod _{C}(\Omega)$ is the modulus of equicontinuity (see Section 2). The $\operatorname{MNC} \varphi$ is monotone, regular, and invariant with respect to union with a compact set.

Proposition 3.6. Under conditions $(\mathscr{B})$ and $(C)$, the multioperator $Q$ is $\varphi$-condensing on bounded subsets of $C([0, T] ; E)$.

Proof. Let $\Omega$ be a bounded subset of $C([0, T] ; E)$ such that

$$
\varphi(Q(\Omega)) \geq \varphi(\Omega)
$$

in the sense of the order generated by the cone $\mathbb{R}_{+}^{2}$.

We estimate the value of $\phi(Q(\Omega))$. Since the operator $\mathscr{C}$ is completely continuous, we need to estimate only

$$
\phi\left((I-\Lambda \mathscr{P}) G \mathscr{P}_{F}(\Omega)\right)
$$

We have

$$
\phi\left(\Lambda \mathscr{B}_{G} \mathscr{P}_{F}(\Omega)\right) \leq\|\Lambda\|^{\left(\chi_{E}, \phi\right)} \chi_{E}\left(\mathscr{S}_{B} \mathscr{P}_{F}(\Omega)\right) \leq\|\Lambda\|^{\left(\chi_{E}, \phi\right)} b \phi\left(G \mathscr{P}_{F}(\Omega)\right)
$$

To estimate $\phi\left(G \mathscr{P}_{F}(\Omega)\right)$, note that

$$
\begin{aligned}
\chi_{E}(U(t-s) F(s, \Omega(s))) & \leq\|U(t-s)\|^{(\chi)} \chi_{E}(F(s, \Omega(s))) \\
& \leq h(t-s) k(s) \chi_{E}(\Omega(s)) \\
& \leq h(t-s) k(s) \phi(\Omega) .
\end{aligned}
$$


Hence, by the theorem on $\chi$-estimates for a multivalued integral (see [8, Theorem 4.2.3]), we have for every $t \in[0, T]$

$$
\chi_{E}\left(G \mathscr{P}_{F}(\Omega)(t)\right) \leq \phi(\Omega) \cdot \int_{0}^{t} h(t-s) k(s) d s
$$

Therefore,

$$
\begin{aligned}
\phi\left(G \mathscr{P}_{F}(\Omega)\right) & \leq \phi(\Omega) \cdot \sup _{0 \leq t \leq T} \int_{0}^{t} h(t-s) k(s) d s, \\
\phi\left[(I-\Lambda \mathscr{B}) G \mathscr{P}_{F}(\Omega)\right] & \leq\left[1+b\|\Lambda\|\left(\chi_{E}, \phi\right)\right] \sup _{0 \leq t \leq T} \int_{0}^{t} h(t-s) k(s) d s \cdot \phi(\Omega) \\
& =\mu \phi(\Omega) .
\end{aligned}
$$

So

$$
\phi(\Omega) \leq \phi(Q(\Omega)) \leq \mu \phi(\Omega)
$$

It follows that $\phi(\Omega)=0$ and hence

$$
\chi_{E}(\Omega(t)) \equiv 0 \quad \text { on }[0, T]
$$

Now, we show that the set $\Omega$ is equicontinuous. Note that from

$$
\bmod _{C}(\Omega) \leq \bmod _{C}(Q(\Omega))
$$

it follows that it is sufficient to show that $Q(\Omega)$ is equicontinuous. It is also sufficient to verify that the set $\left(I-\Lambda \mathscr{B}_{B}\right) G \mathscr{P}_{F}(\Omega)$ is equicontinuous. This is equivalent to show that every sequence $\left\{g_{n}\right\} \subset(I-\Lambda \mathscr{B}) G \mathscr{P}_{F}(\Omega)$ satisfies this property.

Given a sequence $\left\{g_{n}\right\}$, there exist a sequence $\left\{y_{n}\right\} \subset \Omega$ and a sequence of selectors $\left\{f_{n}\right\}, f_{n} \in \mathscr{P}_{F}\left(y_{n}\right)$, such that

$$
g_{n}=(I-\Lambda \mathscr{B}) G\left(f_{n}\right), \quad n=1,2, \ldots
$$

Condition (F3) implies that the sequence of functions $\left\{f_{n}\right\}$ is integrably bounded. The sequence $\left\{y_{n}\right\}$ satisfies the equality

$$
\chi_{E}\left(\left\{y_{n}(t)\right\}\right)=0, \quad \forall t \in[0, T]
$$

hence, by condition (F4), we have

$$
\chi_{E}\left(\left\{f_{n}(t)\right\}\right)=0 \quad \text { for a.a. } t \in[0, T],
$$


and so the sequence $\left\{f_{n}\right\}$ is semicompact (see [8, Definition 4.2.1]). Since the operator $(I-\Lambda \mathscr{B}) G$ satisfies conditions $\left(\mathrm{S}^{\prime}\right)$ and $(\mathrm{S} 2)$, then we can use $[8$, Theorem 5.1.1] to conclude that the sequence $\left\{g_{n}\right\}$ is relatively compact and, consequently, equicontinuous.

Now the relative compactness of $\Omega$ follows from the Ascoli-Arzelà theorem.

We can now observe that it is possible to apply the topological degree theory introduced in Section 2.3 to the multioperator $Q$. We can then formulate the following general existence principle.

Theorem 3.7. Let $W \subset C([0, T] ; E)$ be a bounded open subset such that $x \notin Q(x)$ for all $x \in \partial W$. If $\operatorname{deg}(Q, \bar{W}) \neq 0$, then the set $\sum$ of all mild solutions of problem (3.14) and (3.15) is nonempty.

As a realization of this general principle, we can consider the following situation.

THEOREм 3.8. Under the above conditions, suppose additionally that

(i) there exists a sequence of functions $\omega_{n} \in L_{+}^{1}[0, T], n=1,2, \ldots$, such that

$$
\begin{gathered}
\sup _{\|y\| \leq n}\|F(t, y)\| \leq \omega_{n}(t) \quad \text { for a.a. } t \in[0, T], n=1,2, \ldots, \\
\qquad \underline{\lim _{n \rightarrow \infty}} \frac{1}{n} \int_{0}^{T} \omega_{n}(t) d t=0
\end{gathered}
$$

(ii) the following asymptotic condition holds:

$$
\varliminf_{\|y\| \rightarrow \infty} \frac{\|\mathscr{C} y\|}{\|y\|}=0
$$

Then the set $\Sigma$ of all mild solutions of problem (3.14) and (3.15) is nonempty.

Proof. We prove that there exists a closed ball $B_{r} \subset C([0, T] ; E)$ such that $Q\left(B_{r}\right) \subseteq$ $B_{r}$.

Supposing the contrary, we have the sequences $\left\{y_{n}\right\},\left\{z_{n}\right\} \subset C([0, T], E)$ such that $z_{n} \in Q\left(y_{n}\right),\left\|y_{n}\right\| \leq n$ and $\left\|z_{n}\right\|>n$. Then we obtain

$$
\left\|z_{n}(t)\right\| \leq\left\|\Lambda \mathscr{b} y_{n}\right\|+\left\|G f_{n}(t)\right\|+\left\|\Lambda \mathscr{B} G f_{n}\right\|
$$

for some $f_{n} \in \mathscr{P}_{F}\left(y_{n}\right)$.

Therefore

$$
\left\|z_{n}\right\| \leq\|\Lambda\|\left\|\mathscr{C} y_{n}\right\|+R \int_{0}^{T}\left\|f_{n}(s)\right\| d s+\|\Lambda \mathscr{B}\| R \int_{0}^{T}\left\|f_{n}(s)\right\| d s,
$$

where

$$
R=\sup _{0 \leq t \leq T}\|U(t)\|
$$


Then we have

$$
1 \leq \frac{\left\|z_{n}\right\|}{n} \leq\|\Lambda\| \frac{\left\|\mathscr{C} y_{n}\right\|}{n}+R(1+\|\Lambda \mathscr{B}\|) \frac{1}{n} \int_{0}^{T}\left\|f_{n}(s)\right\| d s .
$$

From (i) and (ii), we see that

$$
\varliminf_{n \rightarrow \infty} \frac{\left\|z_{n}\right\|}{n}=0
$$

giving the contradiction.

It remains only to apply the fixed-point theorem for condensing multimaps (see [8, Corollary 3.3.1]) to the restriction $Q: B_{r} \rightarrow K v\left(B_{r}\right)$.

\subsection{Particular cases}

3.3.1. Case of a constant $c$. Consider a boundary condition of the form

$$
\mathscr{B}(M x)=c
$$

where $c \in E$ and $\mathscr{B}: C([0, T] ; E) \rightarrow E$ is, as before, a bounded linear operator satisfying condition $(\mathscr{B})$. To satisfy condition $(\mathscr{B} \mathscr{C})$, we define the bounded linear operator $\widetilde{\mathscr{B}}: E \rightarrow E$ by

$$
\widetilde{\mathscr{B}} u=\mathscr{B}\left(r_{u}\right)
$$

where $r_{u}(t)=U(t) u$. Then, it is easy to see that condition $(\mathscr{B} \mathscr{C})$ is satisfied if we suppose that

( $\widetilde{\mathscr{B}})$ the operator $\widetilde{\mathscr{B}}$ has inverse $\widetilde{\mathscr{B}}^{-1}$.

In fact, under this condition, we can define the operator $\Lambda$ by the equality

$$
(\Lambda u)(t)=U(t) \cdot \widetilde{\mathscr{B}}^{-1} u
$$

Further, since for every bounded $\Omega \subset E$ we have that

$$
\chi_{E}(\Lambda \Omega(t)) \leq h(t)\left\|\widetilde{\mathscr{B}}^{-1}\right\|^{(\chi)} \chi(\Omega)
$$

and hence

$$
\phi(\Lambda \Omega) \leq\left\|\widetilde{\mathscr{S}}^{-1}\right\|^{(\chi)} \sup _{0 \leq t \leq T} h(t) \cdot \chi(\Omega)
$$

to satisfy condition (C), it is sufficient to suppose that

$$
\left(1+b\left\|\widetilde{\mathscr{S}}^{-1}\right\|^{(\chi)} \sup _{0 \leq t \leq T} h(t)\right) \cdot \sup _{0 \leq t \leq T} \int_{0}^{T} h(t-s) k(s) d s<1 .
$$


3.3.2. Periodic problem. We consider the periodic boundary condition of the form

$$
M x(T)-M x(0)=0
$$

Clearly this is a particular case of the previous problem with

$$
\mathscr{B}_{T} y=y(T)-y(0)
$$

and $c=0$.

Note that from the algebraic semiadditivity property of the Hausdorff MNC, it follows that condition $(\mathscr{B})$ is fulfilled for $\mathscr{B}_{T}$ with the constant $b=2$.

Further, the operator $\widetilde{\mathscr{B}}$ has the form

$$
\widetilde{\mathscr{B}} u=[U(T)-I] u
$$

and condition $(\widetilde{\mathscr{B}})$ is reduced to condition $\left(\widetilde{\mathscr{S}}_{T}\right)$ of the existence of the inverse $[U(T)-I]^{-1}$. The operator $\Lambda$ may be written as

$$
\left(\Lambda_{T} u\right)(t)=U(t)[U(T)-I]^{-1} u
$$

and the integral multioperator $Q$ takes the form

$$
\begin{aligned}
Q_{T}(y)= & (I-\Lambda \mathscr{B}) G \mathscr{P}_{F}(y) \\
= & \left\{z: z(t)=\int_{0}^{T} U(t-s) f(s) d s\right. \\
& \left.+U(t)[I-U(T)]^{-1} \cdot \int_{0}^{T} U(T-s) f(s) d s: f \in \mathscr{P}_{F}(y)\right\}
\end{aligned}
$$

(see $[7,8])$.

Condition (C) can be written as

$\left(C_{T}\right)\left(1+2\left\|[U(T)-I]^{-1}\right\|(\chi) \cdot \sup _{0 \leq t \leq T} h(t)\right) \cdot \sup _{0 \leq t \leq T} h(t-s) k(s) d s<1$.

As a corollary of Theorem 3.8, we obtain the following statement on the existence of a periodic solution.

Theorem 3.9. Under assumptions $\left(\widetilde{\mathscr{S}}_{T}\right)$ and $\left(C_{T}\right)$, Theorem 3.8(i) implies the existence of a mild solution of problem (3.14) and (3.49).

\section{Acknowledgments}

The work of V. Obukhovskii was supported by GNAMPA and RFBR Grants 0201-00189 and 01-01-00425 and by US CRDF-R.F. Ministry of Education Award VZ-010-0. The work of P. Zecca was partially supported by GNAMPA and MIUR. 


\section{References}

[1] R. R. Akhmerov, M. I. Kamenskii, A. S. Potapov, A. E. Rodkina, and B. N. Sadovskiü, Measures of Noncompactness and Condensing Operators, Operator Theory: Advances and Applications, vol. 55, Birkhäuser Verlag, Basel, 1992.

[2] A. Bressan and G. Colombo, Extensions and selections of maps with decomposable values, Studia Math. 90 (1988), no. 1, 69-86.

[3] R. Cross, Multivalued Linear Operators, Monographs and Textbooks in Pure and Applied Mathematics, vol. 213, Marcel Dekker, New York, 1998.

[4] Z. Ding and A. G. Kartsatos, Nonresonance problems for differential inclusions in separable Banach spaces, Proc. Amer. Math. Soc. 124 (1996), no. 8, 2357-2365.

[5] A. Favini and A. Yagi, Degenerate Differential Equations in Banach Spaces, Monographs and Textbooks in Pure and Applied Mathematics, vol. 215, Marcel Dekker, New York, 1999.

[6] V. E. Fedorov (ed.), Sobolev-Type Equations, Chelyabinskij Gosudarstvennyj Universitet, Chelyabinsk, 2002 (Russian).

[7] M. Kamenskii and V. Obukhovskii, Condensing multioperators and periodic solutions of parabolic functional-differential inclusions in Banach spaces, Nonlinear Anal. 20 (1993), no. 7, 781-792.

[8] M. Kamenskii, V. Obukhovskii, and P. Zecca, Condensing Multivalued Maps and Semilinear Differential Inclusions in Banach Spaces, de Gruyter Series in Nonlinear Analysis and Applications, vol. 7, Walter de Gruyter, Berlin, 2001.

[9] D. Kravvaritis and N. S. Papageorgiou, A boundary value problem for a class of evolution inclusions, Comment. Math. Univ. St. Paul. 40 (1991), no. 1, 29-37.

[10] G. Marino, Nonlinear boundary value problems for multivalued differential equations in Banach spaces, Nonlinear Anal. 14 (1990), no. 7, 545-558.

[11] N. S. Papageorgiou, Boundary value problems for evolution inclusions, Comment. Math. Univ. Carolin. 29 (1988), no. 2, 355-363.

[12] B Boundary value problems and periodic solutions for semilinear evolution inclusions, Comment. Math. Univ. Carolin. 35 (1994), no. 2, 325-336.

[13] R. E. Showalter, Monotone Operators in Banach Space and Nonlinear Partial Differential Equations, Mathematical Surveys and Monographs, vol. 49, American Mathematical Society, Rhode Island, 1997.

[14] K. Yosida, Functional Analysis, Die Grundlehren der Mathematischen Wissenschaften, vol. 123, Springer-Verlag, Berlin, 1965.

[15] P. Zecca and P. L. Zezza, Nonlinear boundary value problems in Banach spaces for multivalue differential equations on a noncompact interval, Nonlinear Anal. 3 (1979), no. 3, 347-352.

Valeri Obukhovskii: Department of Mathematics, Voronezh University, 394693 Voronezh, Russia

E-mail address: valerio@org.vrn.ru

Pietro Zecca: Dipartimento di Energetica "Sergio Stecco," Università degli Studi di Firenze, 50139 Firenze, Italy

E-mail address: pzecca@ing.unifi.it 


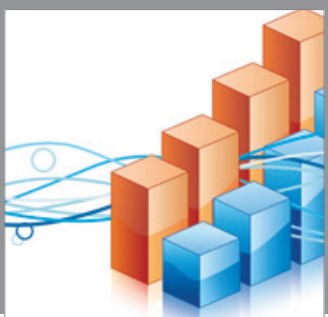

Advances in

Operations Research

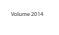

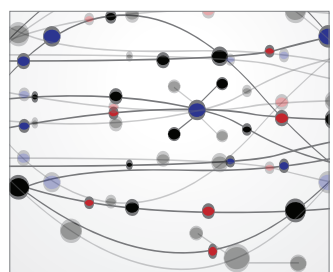

\section{The Scientific} World Journal
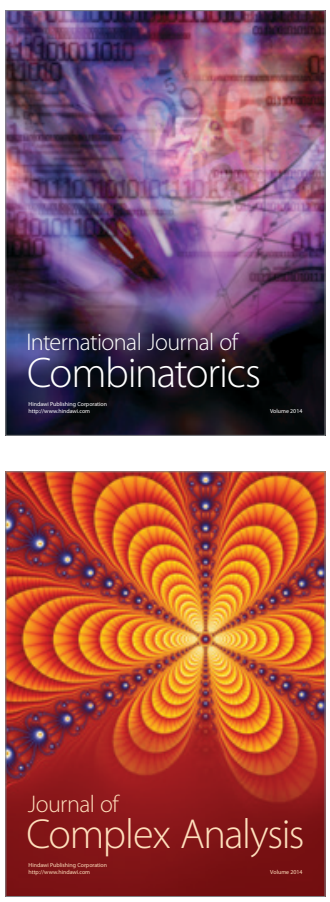

International Journal of

Mathematics and

Mathematical

Sciences
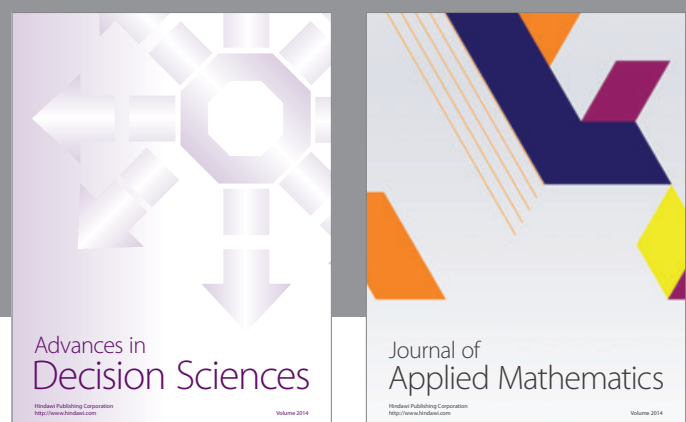

Journal of

Applied Mathematics
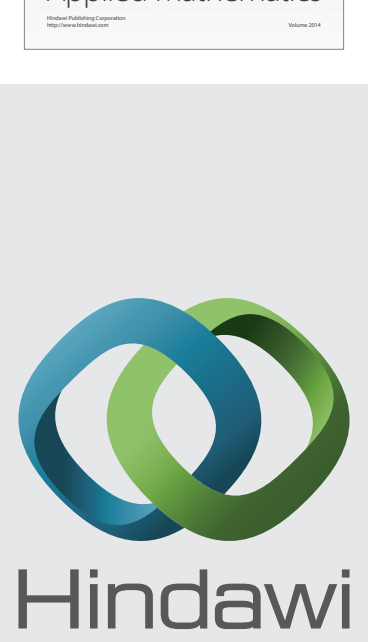

Submit your manuscripts at http://www.hindawi.com
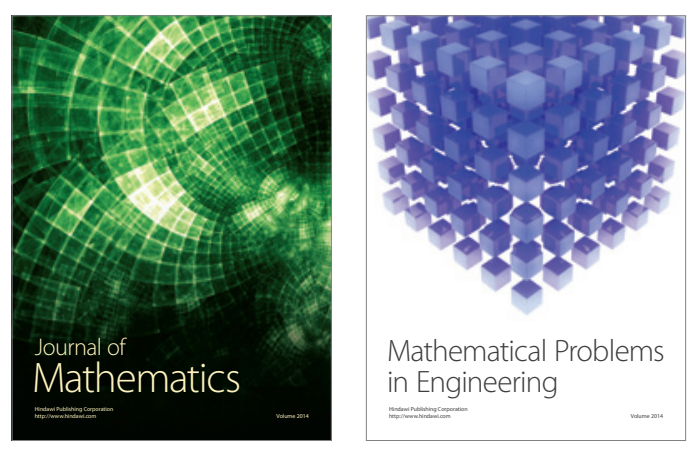

Mathematical Problems in Engineering
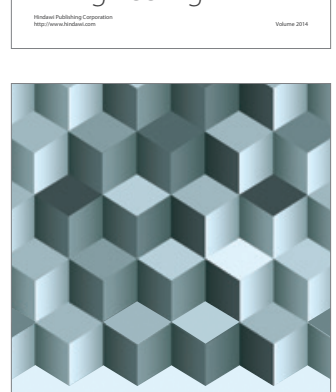

Journal of

Function Spaces
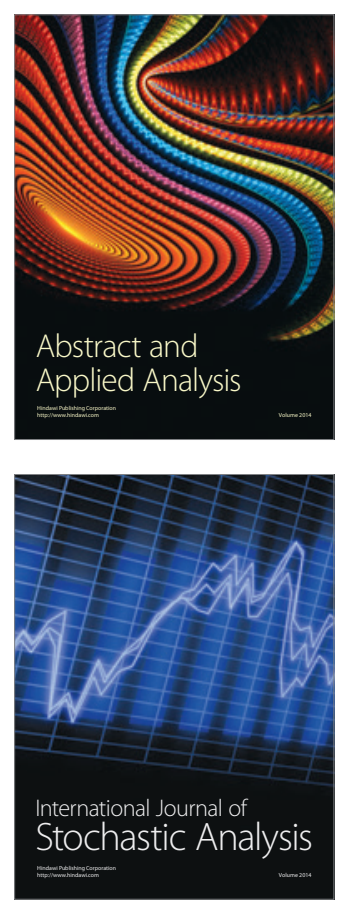

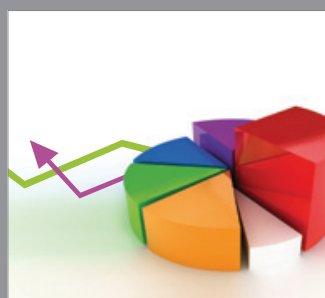

ournal of

Probability and Statistics

Promensencen
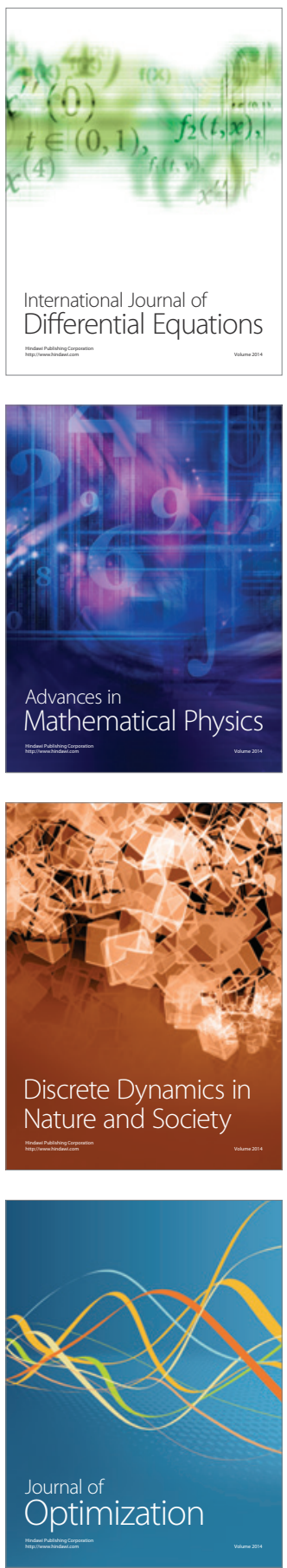